\title{
The Impact of Hepatectomy Time of the Liver Graft on Post-transplant Outcome
}

\author{
A Eurotransplant Cohort Study
}

\author{
Ina Jochmans, MD, PhD, ${ }^{*} \dagger$ Steffen Fieuws, PhD, $\ddagger$ Ineke Tieken, MD, $\S$ \\ Undine Samuel, MD, $\S$ and Jacques Pirenne, $M D, P h D^{*} \dagger$
}

\begin{abstract}
Objective: Assessing the effect of donor hepatectomy time on outcome after transplantation.

Summary of Background Data: When blood supply in a deceased organ donor stops, ischemic injury starts. Livers are cooled to reduce cellular metabolism and minimize ischemic injury. This cooling is slow and livers are lukewarm during hepatectomy, potentially affecting outcome.

Methods: We used the Eurotransplant Registry to investigate the relationship between donor hepatectomy time and post-transplant outcome in 12,974 recipients of deceased-donor livers (January 1, 2004, to December 31, 2013). Cox regression analyses for patient and graft survival (censored and uncensored for death with a functioning graft) were corrected for donor, preservation, and recipient variables. Donor hepatectomy time was defined as time between start of aortic cold flush and placement of the liver in the ice-bowl. Results: Median donor hepatectomy time was 41 minutes [interquartile range (IQR) 32 to 52]. Livers donated after circulatory death had longer hepatectomy times than those from brain-dead donors [50minutes (35 to 68) vs 40 minutes (32 to 51 ), $P<0.001]$. Donor hepatectomy time was independently associated with graft loss [adjusted hazard ratio (HR) 1.03 for every 10minute increase, $95 \%$ confidence interval $(95 \% \mathrm{CI}) 1.02-1.05 ; P<0.001]$. The magnitude of this effect was comparable to the effect of each hour of additional cold ischemia time (adjusted HR 1.04, 95\% CI 1.02-1.05; $P<$ 0.001 ). Donor hepatectomy time had a similar effect on death-censored graft
\end{abstract}

From the *Laboratory of Abdominal Transplant Surgery, Department of Microbiology and Immunology, KU Leuven, Leuven, Belgium; †Abdominal Transplant Surgery, University Hospitals Leuven, Leuven, Belgium; †I-BioStat, Department of Public Health and Primary Care, KU Leuven and Universiteit Hasselt, Leuven, Belgium; and §Eurotransplant International Foundation, Leiden, the Netherlands.

Ina Jochmans did the study design, data interpretation, writing of the manuscript; Steffen Fieuws did the data analyses and writing of the manuscript; Ineke Tieken and Undine Samuel did the data collection and critical reviewing of the manuscript; and Jacques Pirenne did the study design and critical reviewing of the manuscript.

No funding was available for this study.

All authors give final approval of the version to be published and all agree to be accountable for all aspects of the work in ensuring that questions related to the accuracy or integrity of any part of the work are appropriately investigated and resolved.

IJs institution received travel grants from Astellas Pharma, speakers fees from Organ Recovery Systems and Sanofi Genzyme, and consultancy fees from Noorik Biopharmaceuticals.

IJ and JP are holders of a named KU Leuven Chair of the Centrale Afdeling voor Fractionering, Belgium.

JP holds a named KU Leuven Chair of the IGL-group.

The authors disclose no conflicts of interest.

Supplemental digital content is available for this article. Direct URL citations appear in the printed text and are provided in the HTML and PDF versions of this article on the journal's Web site (www.annalsofsurgery.com).

Reprints: Ina Jochmans, MD, PhD, Abdominal Transplant Surgery, University Hospitals Leuven, Herestraat 49, 3000 Leuven, Belgium.

E-mail: ina.jochmans@uzleuven.be.

Copyright (C) 2017 Wolters Kluwer Health, Inc. All rights reserved.

ISSN: 0003-4932/16/XXXX-0001

DOI: $10.1097 /$ SLA.0000000000002593 survival and patient survival. Livers donated after circulatory death and those with a higher donor risk index were more susceptible to the effect of donor hepatectomy time on death-censored graft survival.

Conclusion: Donor hepatectomy time impairs liver transplant outcome. Keeping this time short together with efficient cooling during hepatectomy might improve outcome.

Keywords: donor hepatectomy time, extraction time, graft survival, liver transplantation, organ donation, patient survival, registry analysis, transplant outcome

(Ann Surg 2017;xx:xxx-xxx)

iver transplantation can be lifesaving for patients with acute liver — failure, chronic liver disease, hepatocellular carcinoma, and variant syndromes. ${ }^{1}$ Improved donor and recipient selection and immunosuppression have enhanced short and long-term outcomes compared with the early days of liver transplantation. Current graft and patient survival of $80 \%$ to $90 \%$ at 1 year and $64 \%$ to $85 \%$ at 5 years after transplantation have been reported. ${ }^{2-4}$ Although these outcomes are better than in earlier periods, further improvement would be desirable. Indeed, there is still considerable morbidity and mortality for those listed for transplantation (around 18\%). ${ }^{2}$ Therefore, maximizing graft survival and reducing the need for retransplantation is vital, not only to improve outcome for those who are transplanted but also for those who are waiting for a liver graft.

Ischemic injury, inevitable during procurement and transplantation, increases the risk of primary nonfunction, early allograft dysfunction, and ischemic biliary type strictures and reduces graft and patient survival. ${ }^{5-7}$ Cold storage, currently the gold standard for liver graft preservation, aims to minimize ischemic injury by storing the liver around $4^{\circ} \mathrm{C}$. Basic laws of biochemical kinetics state that metabolism decreases with decreasing temperature, ${ }^{8}$ reducing the effect of ischemia. Cooling the liver in the donor is accomplished by a combination of intravascular and topical cooling. Intravascular cooling consists out of flushing the liver with an ice-cold preservation solution, either through the aorta alone or through both aorta and portal vein. Topical cooling means the organs are covered with ice slush. Despite these maneuvers, the liver cools slowly and liver temperature does not reach $0^{\circ} \mathrm{C}$ to $4^{\circ} \mathrm{C}$ inside the donor body (SDC 1 , Table S1, http://links.lww.com/SLA/B348). ${ }^{9,10}$ Until the liver has cooled down, best achieved by storage on ice, it will continue to experience a higher metabolic rate in an ischemic environment. The time it takes to remove the liver from the donor body, called extraction time or donor hepatectomy time, might be harmful to the graft, as it has not yet reached cold-storage temperatures, and therefore might influence outcome after transplantation. Indeed, a recent report showed that longer kidney extraction times increase the risk of delayed graft function early after kidney transplantation. ${ }^{11}$

The effect of donor hepatectomy time on outcome after liver transplantation has not been investigated. A systematic literature 
search (SDC 1, http://links.lww.com/SLA/B348) could not identify any papers where the role of donor hepatectomy time as a potential factor impacting outcome was studied.

We hypothesized that longer donor hepatectomy times impair graft and patient survival after liver transplantation. We aimed to define this effect and to explore its potential interactions using the Eurotransplant Registry.

\section{METHODS}

\section{Study Population}

Eurotransplant is an international nonprofit organization that manages patient-oriented allocation and cross-border exchange of deceased donor organs in 8 countries: Austria, Belgium, Croatia, Germany, Hungary, Luxembourg, the Netherlands, and Slovenia. The Eurotransplant Registry prospectively records data for all liver transplants performed in 38 liver transplant centers in its region. Data are collected on a voluntary basis to develop best practice recommendations and policies to improve organ allocation and transplant outcomes. $^{2}$ We analyzed data submitted to this registry from all recipients of solitary liver transplants from deceased-donors undertaken between January 1, 2004, and December 31, 2013. The study was approved by the Eurotransplant Liver and Intestine Committee in which all Eurotransplant member states and their scientific organizations are represented.

Donor hepatectomy time was defined as the time between the start of aortic cold flush in the donor to completion of the donor hepatectomy when the liver is placed on ice on the back-table. In case of donation after circulatory death (DCD), in which death is certified following cessation of cardiopulmonary function after withdrawal of life-supporting treatment, ${ }^{12}$ organs undergo a period of warm ischemia. This donor warm ischemia was defined as the time between circulatory arrest in the donor and start of the aortic cold flush. Other data regarding donor warm ischemia time, such as the agonal phase or functional warm ischemia time, were not available for this cohort. Cold ischemia time was defined as the time between the start of the cold flush in the donor and the time when the liver is removed from cold storage on ice and placed in the recipient body for implantation. Patient survival was defined as time from transplantation until death. Graft loss refers to all-cause graft loss, and graft survival was taken as time from transplantation to relisting for liver transplantation or death of the patient. We also studied death-censored graft survival in which case graft loss due to patient death with a functioning graft was censored.

We calculated the Donor Risk Index (DRI) for all transplants as a marker for organ quality. ${ }^{13}$ As the Eurotransplant Registry does not record data on donor ethnicity, we considered all donors not to be of African-American ethnicity. As sharing schemes are different in Eurotransplant compared with those used in the DRI, ${ }^{2}$ we did not take the parameters on regional or national sharing into account.

\section{Statistical Analysis}

Follow-up analysis of the study population included all data submitted to Eurotransplant by May 3, 2016. Only recipients for whom data for both donor hepatectomy time and outcome were available were included in the study. Continuous variables are presented as median (interquartile range, IQR) and categorical variables as number $(\%)$.

Multivariable Cox regression models were used to evaluate the relation of donor hepatectomy time with graft, death-censored graft, and patient survival. Variables in the multivariable models were included if they were shown to affect transplant outcome in scientific literature and were available in the Eurotransplant Registry. In addition, possible confounders that might affect the association between donor hepatectomy time and outcome were considered (Table 1). A multivariable imputation was performed for variables with missing data (SDC 2, http://links.lww.com/SLA/B348). Once the set of confounders was determined based on the backward stepwise selection with multiple imputation, the model was extended with donor hepatectomy time. Furthermore, a random effect of center was added to model the correlation between the survival times of patients within the same center, as the recipient center impacts on outcome. ${ }^{14}$ As simultaneous correction for 2 random effects in Cox regression was not feasible in this dataset, separate analyses were performed correcting for donor and recipient center separately. These analyses indicated that the impact of recipient center on outcome was more important than the effect of donor center (donor and recipient centers can be different, data not shown); therefore, results from the analyses correcting for recipient center are reported. Nevertheless, the effect of donor hepatectomy time on outcome was similar when the Cox regression models were corrected for donor center (data not shown). By including a random effect for recipient center, the interpretation of the effect of donor hepatectomy time refers to differences in risk between patients within the same center having a different length of donor hepatectomy time. ${ }^{15}$ Centers were anonymized in the analyses. The effect of donor hepatectomy time in the multivariable Cox regression model was visualized centering the donor hepatectomy time on the mean of the center to mimic the correction for the random center effect. The result of this visualization was based on 10 imputed datasets, aggregating the results on the log-log scale of the survivor function and the figure gives the mean survival function for varying values of donor hepatectomy time, adjusted for all other covariates in the Cox model. ${ }^{16}$ Note, results are based on the assumption that time to graft loss and time to death with a functioning graft are not related.

A graphical procedure was used to verify that the effect of donor hepatectomy time was constant over time (data not shown) and donor hepatectomy time was used as a fixed effect in the models. ${ }^{17}$

We performed interaction analyses to determine whether the effect of donor hepatectomy time on outcome was more pronounced in DCD livers versus livers donated after brain death (DBD), livers from donors with a higher DRI, livers with longer cold ischemia times, and whether the effect of donor hepatectomy time was modified by type of the graft (whole graft vs split graft).

$P$ values $<0.050$ were considered significant. All reported results involving variables with missing values were based on multiple imputation. All analyses were performed using SAS software (v9.4 for Windows, SAS Institute nv/sa, Tervuren, Belgium). Reported hazard ratios for donor hepatectomy time refer to a difference of 10 minutes.

\section{RESULTS}

\section{Characteristics of the Study Population}

Fifteen thousand one hundred thirty-six deceased-donor liver transplants were performed in the Eurotransplant region between January 1, 2004, and December 31, 2013. Data on donor hepatectomy time were available in 13,729 cases from which we excluded 330 transplants in which donor hepatectomy times were reported to be extremely short ( $<10$ minutes) or long ( $>200$ minutes). These limits were based upon donor hepatectomy times ( 35 minutes, IQR 24 to 46; min-max: 11 to 103) of 705 livers transplanted in our center between 2000 and 2013. Another 425 cases were excluded because of missing outcome data. Transplant characteristics were comparable between the 12,974 transplants included and the 2162 transplants excluded in this study (Table S2, http://links.lww.com/SLA/B348).

All patients without an event have a minimal follow-up of at least 1 year. Median follow-up after transplantation was 4.0 years (2.4 to 6.2). Table 1 summarizes the donor and recipient characteristics. Median donor hepatectomy time in the Eurotransplant cohort 


\begin{tabular}{|c|c|c|}
\hline Characteristic & Result & $\%$ Missing \\
\hline \multicolumn{3}{|l|}{ Donor } \\
\hline Age, yrs & $51(39-63)$ & 0 \\
\hline Sex & & 0 \\
\hline Male & $6826(53 \%)$ & \\
\hline Female & $6148(47 \%)$ & \\
\hline Body mass index, $\mathrm{kg} / \mathrm{m}^{2}$ & $24.9(22.9-27.6)$ & 0.008 \\
\hline Cause of death & & 0 \\
\hline Trauma & $2851(22 \%)$ & \\
\hline CVA & $7995(62 \%)$ & \\
\hline Anoxia & $1469(11 \%)$ & \\
\hline Other & $659(5 \%)$ & \\
\hline Donor type & & 0 \\
\hline DBD & $12,513(96 \%)$ & \\
\hline DCD & $461(4 \%)$ & \\
\hline \multicolumn{3}{|l|}{ Donor warm ischemia time, min } \\
\hline In DCD transplants & $14(10-19)$ & 0.27 \\
\hline Donor cardiac arrest & & 55.1 \\
\hline Yes & $1289(22 \%)$ & \\
\hline No & $4530(78 \%)$ & \\
\hline \multicolumn{3}{|l|}{ AST, U/L } \\
\hline Highest & $55(32-109)$ & 1.1 \\
\hline Terminal & $45(28-80)$ & 1.1 \\
\hline \multicolumn{3}{|l|}{ Bilirubin, $\mathrm{mg} / \mathrm{dL}$} \\
\hline Highest & $0.6(0.4-1.0)$ & 2.4 \\
\hline Terminal & $0.5(0.3-0.9)$ & 2.4 \\
\hline \multicolumn{3}{|l|}{ Sodium, $\mathrm{mmol} / \mathrm{L}$} \\
\hline Highest & $149(144-155)$ & 0.3 \\
\hline Terminal & $147(142-152)$ & 0.3 \\
\hline Other organs donated & & 0 \\
\hline Heart & $5058(39 \%)$ & \\
\hline Lungs & $3841(30 \%)$ & \\
\hline Heart and lungs & $2483(19 \%)$ & \\
\hline Pancreas & $3780(29 \%)$ & \\
\hline Kidney & $12,125(93 \%)$ & \\
\hline \multicolumn{3}{|l|}{ Recipient } \\
\hline Age, yrs & $53(44-60)$ & 0 \\
\hline Sex & & 0 \\
\hline Male & $8484(65 \%)$ & \\
\hline Female & $4490(35 \%)$ & \\
\hline Body mass index, $\mathrm{kg} / \mathrm{m}^{2}$ & $25.0(22.6-28.4)$ & 0.08 \\
\hline Lab MELD & $18(11-30)$ & 15.2 \\
\hline Indication for transplant & & 0 \\
\hline Acute liver failure & $1045(8 \%)$ & \\
\hline Cholestatic disease & $1406(11 \%)$ & \\
\hline Hepatocellular carcinoma & $1947(15 \%)$ & \\
\hline Postalcoholic cirrhosis & $2065(16 \%)$ & \\
\hline Viral hepatitis & $1574(12 \%)$ & \\
\hline Retransplant & $1808(14 \%)$ & \\
\hline Other & $3218(24 \%)$ & \\
\hline \multicolumn{3}{|l|}{ Process } \\
\hline Preservation fluid & & 0.67 \\
\hline HTK & $8779(68 \%)$ & \\
\hline UW & $3984(31 \%)$ & \\
\hline Other & $124(1 \%)$ & \\
\hline Type of graft & & 0 \\
\hline Whole & $12,054(93 \%)$ & \\
\hline Split & $920(7 \%)$ & \\
\hline Arterial anatomy* & & 68.2 \\
\hline Normal & $4263(74 \%)$ & \\
\hline Abnormal & $153(26 \%)$ & \\
\hline Cold ischemia time, $\mathrm{h}$ & $9.0(7.2-11.0)$ & 38.9 \\
\hline Donor hepatectomy time, min & $41(32-52)$ & 0 \\
\hline
\end{tabular}

Values are presented as median (interquartile range) or number (\%).

AST indicates aspartate aminotransferase; CVA, cerebrovascular accident; DBD, donation after brain death; DCD, donation after circulatory determination of death; HTK, histidine-tryptophan-ketoglutarate solution; MELD, model of end-stage liver disease; UW, University of Wisconsin solution.

${ }^{*}$ As reported by donor surgeon. was 41 minutes (32 to 52) (Fig S1, http://links.lww.com/SLA/B348, histogram) and median cold ischemia time was 9.0 hours $(7.2$ to 11.0). The DRI was 1.5 (1.3 to 1.8 ).

\section{Donor Hepatectomy Time Independently Impairs Outcome}

Donor hepatectomy time increased the overall graft failure rate for all deceased-donor livers [adjusted hazard ratio (HR) 1.03, $95 \%$ confidence interval $(95 \% \mathrm{CI}) 1.02-1.05, P<0.001]$ (Tables 2 and 3). The magnitude of the effect of every 10-minute increase in donor hepatectomy time was comparable to the effect of each hour of additional cold ischemia time (HR 1.04, 95\% CI 1.02-1.05; $P<$ 0.001). Because donor hepatectomy time is an integral part of cold ischemia time, we checked the correlation between both. This correlation was poor (Spearman rho $0.06, P<0.001$ ) and unlikely to confound the results. Indeed, when the analysis was repeated with cold ischemia time minus donor hepatectomy time, the results were the same (Table S3, http://links.lww.com/SLA/B348). Donor hepatectomy time was also an independent risk factor for death-censored graft loss (HR 1.04, 95\% CI 1.01-1.06, $P=0.005$ ) and patient death (HR 1.03, 95\% CI 1.01-1.05, $P=0.001$ ) (Table 2, S4, S5, http:// links.lww.com/SLA/B348).

Outcomes were compromised by donor and recipient age, cold ischemia time, split graft, and indication for transplantation (Tables 3, S4, S5, http://links.lww.com/SLA/B348). DCD donation was an independent risk factor for graft loss and death-censored graft loss but not for patient death (Tables 3, S4, S5, http://links.lww.com/ SLA/B348). Donor terminal sodium concentrations were an independent risk factor for graft loss and death-censored graft loss but not patient death (Tables 3, S4, S5, http://links.lww.com/SLA/B348). The Model of End Stage Liver Disease (MELD) ${ }^{18}$ score was a risk factor for graft loss and patient death but not death-censored graft loss (Tables 3, S4, S5, http://links.lww.com/SLA/B348). Type of preservation fluid had no independent effect on outcome. The effect of increasing donor hepatectomy time on predicted graft loss, adjusted for all other covariables in the Cox model, is visualized in Fig. 1.

As organs are procured in a specific order (heart followed by lungs, liver and/or pancreas, kidneys), liver donor hepatectomy time might be lengthened by procurement of other organs. Hearts, lungs, and both heart and lungs were procured in 39\%, 30\%, and 19\% of cases. The pancreas was procured in $29 \%$ of cases (Table 1). Donor hepatectomy time was indeed longer when hearts [ 45 minutes ( 35 to 57 ) vs 39 minutes ( 30 to 48 ), $P<0.001$ ], lungs [51 minutes ( 42 to 62 ) vs 37 minutes ( 30 to 46 ), $P<0.001$ ], heart and lungs [53 minutes (44 to 64 ) versus 38 minutes (30 to 48 ), $P<0.001$ ], and pancreas (49 minutes ( 38 to 61 ) versus 39 minutes ( 30 to 48 ), $P<0.001$ ] were procured. Only lung procurement was found to be an independent (protective) predictor of graft, death-censored graft, and patient survival (Tables 3, S4, S5, http://links.lww.com/SLA/B348). Univariable analyses of donor factors of lung versus no-lung donors are given in Table S6, http://links.lww.com/SLA/B348.

\section{DCD Grafts are More Susceptible to Donor Hepatectomy Time}

DCD livers had longer donor hepatectomy times than DBD livers [50 minutes (35 to 68 ) vs 40 minutes (32 to 51 ), $P<0.001$ ]. Table S7, http://links.lww.com/SLA/B348 shows demographics of DCD versus DBD transplants; DCD donors were more often male, younger with a lower body mass index, and died more frequently after trauma or anoxia than DBD donors. Donor sodium levels were lower in DCDs. Recipients were older and had lower MELD scores. DCD grafts were rarely used for acute liver failure or retransplantation. 
TABLE 2. Hazard Ratio of Donor Hepatectomy Time* in Cox Regression Models for Graft Failure, Patient Death, and Deathcensored Graft Loss After Liver Transplantation

\begin{tabular}{|c|c|c|c|c|}
\hline & \multicolumn{2}{|c|}{ Unadjusted } & \multicolumn{2}{|c|}{ Adjusted } \\
\hline & HR $(95 \%$ CI $)$ & $P$ & HR $(95 \%$ CI $)$ & $P$ \\
\hline Death-censored Graft survival & $1.02(1.00-1.04)$ & 0.080 & $1.04(1.01-1.06)$ & 0.005 \\
\hline Graft survival & $1.02(1.00-1.03)$ & 0.030 & $1.03(1.02-1.05)$ & $<0.001$ \\
\hline
\end{tabular}

CI indicates confidence interval; HR, hazard ratio.

* $10 \mathrm{~min}$.

†HR and $P$ values of the other covariables considered in the model are provided in Tables 3 and 4 .

DCD donation was an independent predictor of graft loss (HR $1.60,95 \%$ CI $1.35-1.90, P<0.001)$ and death-censored graft loss (HR 2.21, 95\% CI 1.76-2.78, $P<0.001$ ) but not patient survival.

We assessed whether the detrimental effect of donor hepatectomy time was more pronounced in DCD than DBD livers and therefore examined the interaction effect between DCD and DBD livers and donor hepatectomy time in the multivariable models. The effect of donor hepatectomy time on death-censored graft loss was more pronounced in recipients of DCD livers than in recipients of DBD livers (HR 1.14, 95\% CI 1.06-1.22 for DCD, $1.03,95 \% \mathrm{CI}$ 1.00-1.05 for DBD, $P=0.008$ for interaction), but this was not the case for graft loss or patient death (Table 4). Figure 2 visualizes the effect of increasing donor hepatectomy time on predicted

TABLE 3. Results from an Additive Multivariable Cox Regression Model for Graft Loss After Liver Transplantation Obtained After Backward Stepwise Selection Based on Multiple Imputations

\begin{tabular}{lcr}
\hline & Hazard Ratio (95\% CI) & \multicolumn{1}{c}{$\boldsymbol{P}$} \\
\hline Donor Hepatectomy Time (10 min) & $1.03(1.02-1.05)$ & $<0.001$ \\
Donor age, yrs & $1.01(1.01-1.01)$ & $<0.001$ \\
Lungs retrieved (Y vs N) & $0.91(0.85-0.97)$ & 0.004 \\
Donor last sodium, mmol/L & $1.00(1.00-1.01)$ & 0.011 \\
Donor cardiac arrest (Y vs N) & $0.93(0.84-1.04)$ & 0.224 \\
DCD vs DBD & $1.60(1.35-1.90)$ & $<0.001$ \\
Cold ischemia time, h & $1.04(1.02-1.05)$ & $<0.001$ \\
Recipient body mass index & $0.99(0.98-1.00)$ & 0.003 \\
Recipient age, yrs & $1.01(1.01-1.01)$ & $<0.001$ \\
Whole vs split liver graft & $0.70(0.62-0.79)$ & $<0.001$ \\
Lab MELD & $1.01(1.01-1.02)$ & $<0.001$ \\
Donor highest AST, U/L (log2) & $1.03(1.01-1.06)$ & 0.009 \\
Donor cause of death & $*$ & 0.113 \\
$\quad$ Trauma & $1.03(0.96-1.11)$ & \\
Cerebrovascular accident & $1.01(0.89-1.15)$ & 0.471 \\
Anoxia & $1.18(1.03-1.35)$ & 0.878 \\
Other & $*$ & 0.014 \\
Indication for transplantation & $1.70(1.51-1.92)$ & $<0.001$ \\
$\quad$ Alcoholic cirrhosis & $1.14(1.02-1.28)$ & 0.001 \\
Acute liver failure & $1.19(1.06-1.34)$ & 0.003 \\
Hepatocellular carcinoma & $2.04(1.85-2.26)$ & $<0.001$ \\
Cholestatic disease & $1.27(1.14-1.41)$ & $<0.001$ \\
Retransplant & $1.12(1.02-1.23)$ & 0.019 \\
Viral hepatitis & & \\
Other &
\end{tabular}

The result is based on 5412 events from 12,974 subjects. After model reduction, donor hepatectomy time was added as a predictor and recipient center was added as a random effect.

AST indicates aspartate aminotransferase; CI, confidence interval; DBD, donation after brain death; DCD, donation after circulatory death; MELD, model for end-stage liver disease.

*Reference category. death-censored graft survival in the first year after transplantation, adjusted for all other covariables in the Cox model. If a center accepted a DCD liver with a donor hepatectomy time 30 minutes longer than the mean donor hepatectomy time in that center, the risk for death-censored graft loss 1 year after transplantation increased by $8.7 \%$ (from $23.1 \%$ to $31.8 \%$ ), and for DBD livers, the risk increased by $1.2 \%$ (from $14.5 \%$ to $15.7 \%$ ).

\section{Higher Risk Grafts are also More Susceptible to Donor Hepatectomy Time}

We evaluated whether prolonged cold ischemia time, graft quality (assessed by DRI), or type of graft (whole vs split) might affect the susceptibility of the graft to increased donor hepatectomy time. Interaction effects between donor hepatectomy time and cold ischemia time, DRI, and type of graft were investigated separately in the multivariable model for graft, death-censored graft, and patient, survival. Although cold ischemia time and type of graft were independently associated with outcome, the unfavorable effect of prolonged donor hepatectomy time on survival was not influenced by either in any of the multivariable models (data not shown).

The effect of donor hepatectomy time on graft and deathcensored graft survival-but not patient survival-was more pronounced in livers with a higher DRI (HR 1.10, 95\% CI 1.01-1.19, $P$ $=0.024$ for interaction and HR $1.06,95 \%$ CI $1.00-1.13, P=0.044$ for interaction, respectively).

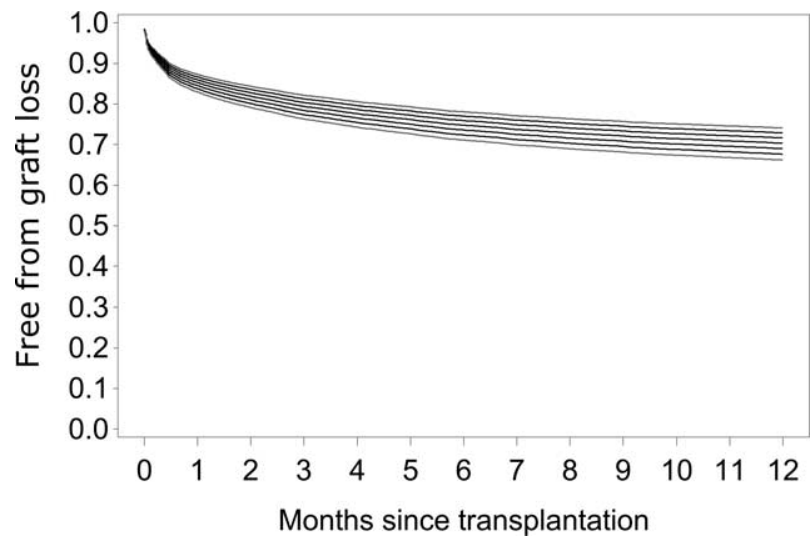

FIGURE 1. Predicted graft survival function during the first 12 months post-transplant for selected values of donor hepatectomy time [ie, 30 min below center mean (top line), 60 min above the center mean (bottom line), and intermediate $15 \mathrm{~min}$ incremental values (middle lines)], adjusted for all other covariables in the Cox model. 
TABLE 4. Donor Type (DBD/DCD) and the Interaction with Donor Hepatectomy Time (10 min) is Added to the Multivariable Cox Regression for Graft, Death-Censored Graft, and Patient Survival

\begin{tabular}{|c|c|c|c|c|c|c|}
\hline & \multicolumn{2}{|c|}{ Graft Survival } & \multicolumn{2}{|c|}{ Death-Censored Graft Survival } & \multicolumn{2}{|c|}{ Patient Survival } \\
\hline & HR $(95 \%$ CI) & $P$ & HR $(95 \%$ CI $)$ & $P$ & HR $(95 \%$ CI $)$ & $P$ \\
\hline Effect when DCD & $1.08(1.02-1.14)$ & 0.008 & $1.14(1.06-1.22)$ & $<0.001$ & $1.05(0.98-1.13)$ & 0.200 \\
\hline Effect when DBD & $1.03(1.01-1.05)$ & $<0.001$ & $1.03(1.00-1.05)$ & 0.055 & $1.03(1.01-1.05)$ & 0.005 \\
\hline
\end{tabular}

Results for the other predictors are not shown.

CI indicates confidence interval; DBD, donation after brain death; DCD, donation after circulatory death; ET, donor hepatectomy time (10 min); HR, hazard ratio.

\section{DISCUSSION}

This analysis of 12,974 deceased donor liver transplants captured in the Eurotransplant Registry shows that the donor hepatectomy time - that is, the time interval between aortic cold flush and the end of donor hepatectomy-increases the risk of graft loss, deathcensored graft loss, and patient death. Every 10-minute increase in donor hepatectomy time had a detrimental effect on outcome similar to that of every hour increase in cold ischemia time. DCD grafts and those with a higher DRI are more vulnerable to the detrimental effect of donor hepatectomy time on death-censored graft survival.

During procurement and before actual cold storage in the ice box, the liver is exposed to potentially deleterious subnormothermic temperatures. In a pig model, studying temperature changes during the procurement procedure, it took 75 to 100 minutes for the liver core temperature to drop below $5^{\circ} \mathrm{C} .^{9}$ A stable temperature of $1^{\circ} \mathrm{C}$ was only reached more than 3 hours after the initiation of the cold flush in the donor body, when the liver had been procured and stored on ice. ${ }^{9}$ Data on human livers are scarce but point to the same findings of a relatively slow decline in temperature as long as the liver has not been stored in the ice box (Table S1, http://links.lww.com/SLA/B348) ${ }^{9,10}$ As far as we are aware, this is the first report on the deleterious effect of this time period.

Liver donor hepatectomy time was longer in DCD donors and, in addition, DCD liver grafts were more susceptible to the detrimental effect of donor hepatectomy time resulting in a higher risk of death-censored graft loss (ie, death because of graft failure or listing for retransplantation). In DCD, unlike in DBD, the entire liver dissection is done after the aortic cross clamp and so the prolonged

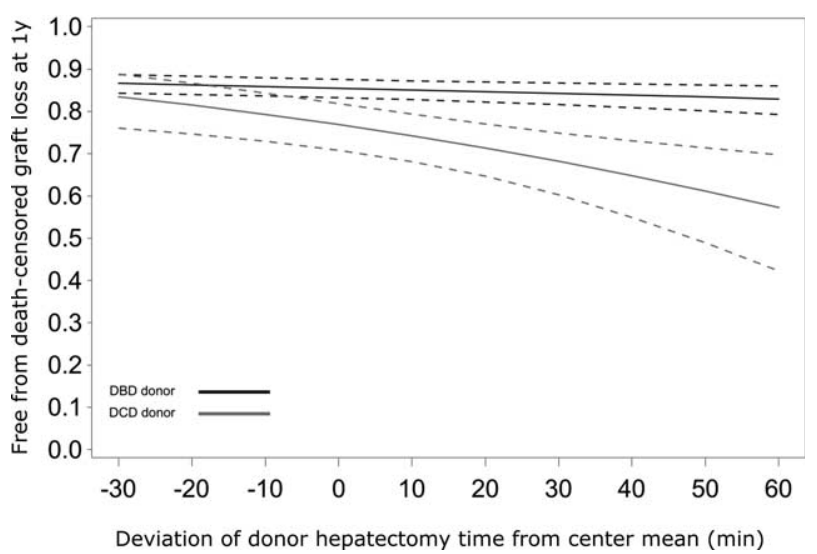

FIGURE 2. Predicted death-censored graft loss within the first year in function of donor hepatectomy time, adjusted for all other covariables in the Cox regression model. Donor hepatectomy time was centered on the mean of the center to mimic the withincenter effect. DBD donor, top line; DCD donor, bottom line. donor hepatectomy time is not unexpected. Indeed, in DCD donation, a "super-rapid" procurement technique is used in which the aortic cold flush is started within minutes after laparotomy, while in the classic procurement technique, much more preparatory dissection is done before aortic cold flushing. ${ }^{19,20}$ The increased vulnerability to donor hepatectomy time could be due to the superimposed preceding exposure of DCD livers to normothermic ischemia starting at withdrawal of therapy, a phase known to cause significant changes at the cellular and subcellular level. ${ }^{21}$

The finding that donor hepatectomy time impairs outcome is of particular importance, as it is a factor amenable to improvement by reducing the duration of donor hepatectomy and improving the efficiency of liver cooling. Although we cannot investigate the effect of the surgeon's experience in this dataset, it would be reasonable to assume that more experienced surgeons are likely to perform the hepatectomy faster. In laparoscopic donor nephrectomy, for example, operative times decrease with increasing surgical experience. ${ }^{22}$ Therefore, our findings support the importance of specific training in procurement surgery and the performance of a critical number of procedures under supervision before performing the procedure independently. ${ }^{23}$ This is particularly true in DCD procedures where DCD livers suffer more surgical injury during procurement than DBD livers, ${ }^{24,25}$ donor hepatectomy times are longer, and DCD livers are more susceptible to its effect. More attention should also be paid to keeping the liver cold during its procurement. Portal-in addition to aortic-cold flush accelerates liver cooling. ${ }^{26,27}$ Available data analyzing aortic versus dual perfusion are contradictory, however, and perhaps merit revisiting in the light of our new findings. ${ }^{27-29}$ Aortic flush is usually stopped after a certain volume of cold preservation solution has been perfused. Whether cold perfusion should be continued until completion of the hepatectomy should be studied. Finally, after hepatectomy, the liver should be transferred to the back-table and the ice box as quickly as possible.

The strength of our analysis is the use of a large cohort of transplant recipients in the Eurotransplant region. Like any registry study, it assumes a similar overall effect of donor hepatectomy time in all centers. A limitation inherent to registry studies based on data from many different centers and countries is the lack of detailed information regarding donor and recipient characteristics and incomplete data registration. In contrast to the USA and UK transplant registries, data submission to the Eurotransplant Registry is not compulsory, ${ }^{2}$ explaining the frequency of missing data in this registry. However, even with mandatory data submission, the final cohort of a recent study looking at implantation time in kidney transplantation, using the United Network of Organ Sharing registry, represented only $57.7 \%$ of the eligible cohort. ${ }^{30}$ Also, as baseline characteristics of transplants excluded because of missing data were comparable to the transplants included, we do not suspect that our results were importantly confounded, although bias cannot entirely be excluded. Multiple imputations were used to reduce any bias caused by missing data as much as possible. Although this large 
cohort study allowed us to perform survival analyses, no in-depth analysis on early outcome variables was possible. A detailed analysis looking at an association of donor hepatectomy time with primary nonfunction, early allograft dysfunction, and the development of biliary complications would be very valuable. As the Eurotransplant Registry does not reliably record these outcomes, further studies in other datasets are needed. In addition, these would allow confirmation of our findings and might allow to shed some light on additional confounding factors such as surgical technique and surgical experience, the type of organ flush (dual cooling via aorta and portal system or aortic cooling only), in situ or ex situ splitting of the liver, etc, that are not detailed in the Eurotransplant Registry.

In conclusion, longer hepatectomy time during donor surgery impairs outcome early after liver transplantation. Livers from DCD and higher risk donors are particularly vulnerable to this detrimental effect. Efforts should be made to perform the donor hepatectomy swiftly and diligently and to cool the liver.

\section{ACKNOWLEDGMENTS}

We thank all centers within Eurotransplant for providing data to the Eurotransplant Registry and Erwin de Vries from Eurotransplant for compilation of the data. We also thank the Eurotransplant Liver and Intestinal Advisory Committee and the Eurotransplant Organ Procurement Committee for their support.

\section{REFERENCES}

1. Rashid ST, Gimson A. General considerations. In: Neuberger J, Ferguson JW, Newsome PN, eds. Liver Transplantation: Clinical Assessment and Management. Oxford, UK: Wiley; 2013:3-17.

2. Jochmans I, van Rosmalen M, Pirenne J, et al. Adult liver allocation in Eurotransplant. Transplantation. 2017;101:1542-1550.

3. NHS Blood and Transplant, Annual Report on Liver Transplantation, report for for 2015/2016. NHSBT, http://www.odt.nhs.uk/pdf/organ_specific_report_ liver_2016.pdf. Accessed February 6, 2017.

4. Kim WR, Lake JR, Smith JM, et al. OPTN/SRTR 2015 annual data report: liver. Am J Transplant. 2017;17(Suppl 1):174-251.

5. Ali JM, Davies SE, Brais RJ, et al. Analysis of ischemia/reperfusion injury in time-zero biopsies predicts liver allograft outcomes. Liver Transpl. 2015;21:487-499.

6. Feng S, Goodrich NP, Bragg-Gresham JL, et al. Characteristics associated with liver graft failure: the concept of a donor risk index. Am J Transplant. 2006;6:783-790.

7. Rana A, Hardy MA, Halazun KJ, et al. Survival outcomes following liver transplantation (SOFT) score: a novel method to predict patient survival following liver transplantation. Am J Transplant. 2008;8:2537-2546.

8. Gillooly JF, Brown JH, West GB, et al. Effects of size and temperature on metabolic rate. Science. 2001;293:2248-2251.

9. Hertl M, Howard TK, Lowell JA, et al. Changes in liver core temperature during preservation and rewarming in human and porcine liver allografts. Liver Transpl Surg. 1996;2:111-117.
10. Villa R, Fondevila C, Erill I, et al. Real-time direct measurement of human liver allograft temperature from recovery to transplantation. Transplantation. 2006;81:483-486.

11. Osband AJ, James NT, Segev DL. Extraction time of kidneys from deceased donors and impact on outcomes. Am J Transplant. 2016;16:700-703.

12. Kootstra G, Daemen JH, Oomen AP. Categories of non-heart-beating donors. Transplant Proc. 1995;27:2893-2894.

13. Schaubel DE, Sima CS, Goodrich NP, et al. The survival benefit of deceased donor liver transplantation as a function of candidate disease severity and donor quality. Am J Transplant. 2008;8:419-425.

14. Asrani SK, Kim WR, Edwards EB, et al. Impact of the center on graft failure after liver transplantation. Liver Transpl. 2013;19:957-964.

15. Neuhaus JM, Kalbfleisch JD. Between- and within-cluster covariate effects in the analysis of clustered data. Biometrics. 1998;54:638-645.

16. Makuch RW. Adjusted survival curve estimation using covariates. J Chronic Dis. 1982;35:437-443.

17. Lin DY, Wei LJ, Ying Z. Checking the Cox model with cumulative sums of martingale-based residuals. Biometrika. 1993;80:557-572.

18. Malinchoc M, Kamath PS, Gordon FD, et al. A model to predict poor survival in patients undergoing transjugular intrahepatic portosystemic shunts. Hepatology. 2000;31:864-871.

19. Casavilla A, Ramirez C, Shapiro R, et al. Experience with liver and kidney allografts from non-heart-beating donors. Transplantation. 1995;59:197-203.

20. Starzl TE, Hakala TR, Shaw BW Jr, et al. A flexible procedure for multiple cadaveric organ procurement. Surg Gynecol Obstet. 1984;158:223-230.

21. Rhee JY, Alroy J, Freeman RB. Characterization of the withdrawal phase in a porcine donation after the cardiac death model. Am J Transplant. 2011;11:1169-1175.

22. Raque J, Billeter AT, Lucich E, et al. Training techniques in laparoscopic donor nephrectomy: a systematic review. Clin Transplant. 2015;29:893-903.

23. de Graauw JA, Mihaly S, Deme O, et al. Exchange of best practices within the European Union: surgery standardization of abdominal organ retrieval. Transplant Proc. 2014;46:2070-2074.

24. Ausania F, White SA, Coates R, et al. Liver damage during organ donor procurement in donation after circulatory death compared with donation after brain death. Br J Surg. 2013;100:381-386.

25. de Boer JD, Kopp WH, Ooms K, et al. Abdominal organ procurement in the Netherlands: an analysis of quality and clinical impact. Transpl Int. 2017;30:288-294.

26. Chui AK, Thompson JF, Lam D, et al. Cadaveric liver procurement using aortic perfusion only. Aust N Z J Surg. 1998;68:275-277.

27. D'Amico F, Vitale A, Gringeri E, et al. Liver transplantation using suboptimal grafts: impact of donor harvesting technique. Liver Transpl. 2007;13: 1444-1450.

28. Brockmann JG, Vaidya A, Reddy S, et al. Retrieval of abdominal organs for transplantation. Br J Surg. 2006;93:133-146.

29. NHS Blood and Transplant Organ Donation and Transplantation Directorate Clinical Retrieval Group. Abdominal Perfusion and Preservation Protocols for NORS Teams in the UK version 1.9, 2015. http://www.odt.nhs.uk/pdf/Abdominal_Perfusion_Protocol.pdf. Accessed February 6, 2017.

30. Tennankore KK, Kim SJ, Alwayn IP, et al. Prolonged warm ischemia time is associated with graft failure and mortality after kidney transplantation. Kidney Int. 2016;89:648-658. 